\title{
E-Cadherin fragments as potential mediators for peritoneal metastasis in advanced epithelial ovarian cancer
}

\author{
Fabian Trillsch ${ }^{1,5}$, Sascha Kuerti ${ }^{1,5}$, Christine Eulenburg ${ }^{2}$, Eike Burandt ${ }^{3}$, Linn Woelber ${ }^{1}$, Katharina Prieske ${ }^{1}$, \\ Kathrin Eylmann ${ }^{1}$, Leticia Oliveira-Ferrer ${ }^{1}$, Karin Milde-Langosch ${ }^{1}$ and Sven Mahner ${ }^{\star}, 1,4$ \\ ${ }^{1}$ Department of Gynaecology and Gynaecologic Oncology, University Medical Centre Hamburg-Eppendorf, Martinistr 52, Hamburg \\ 20246, Germany; ${ }^{2}$ Department of Medical Biometry and Epidemiology, University Medical Centre Hamburg-Eppendorf, Martinistr 52, \\ Hamburg 20246, Germany; ${ }^{3}$ Institute of Pathology, University Medical Centre Hamburg-Eppendorf, Martinistr 52, Hamburg 20246, \\ Germany and ${ }^{4}$ Department of Obstetrics and Gynaecology, University of Munich, Marchioninistr 15, Munich 81377, Germany
}

Background: Peritoneal dissemination and retroperitoneal lymph node involvement are main routes for tumour spread of epithelial ovarian cancer (EOC), possibly determined by the intercellular connecting protein E-Cadherin (E-Cad) and its fragments.

\begin{abstract}
Methods: Tumour tissue of 105 advanced EOC patients was evaluated for protein expression of E-Cad, $\beta$-Catenin and Calpain by western blotting and immunohistochemistry. Expression patterns were compared between tumours with solely intraperitoneal ( $p T 3 c, p N O ; n=41$ ) and tumours with retroperitoneal metastases ( $\mathrm{pT} 1 \mathrm{a}-3 \mathrm{c}, \mathrm{pN} 1 ; n=64)$. Lysates of the EOC cell line SKOV3 and tumour tissue from the intraperitoneal group were tested for E-Cad expression following Calpain treatment.
\end{abstract}

Results: E-Cad full-length (E-Cad-FL, $120 \mathrm{kDa}$ ) and two major fragments at $85 \mathrm{kDa}$ (E-Cad-85) and $23 \mathrm{kDa}$ (E-Cad-23) were detected by western blotting. E-Cad-85 expression was significantly higher in tumours with solely intraperitoneal metastases and correlated strongly with E-Cad-23 and the protease Calpain. Calpain-mediated cleavage was identified as a potential mechanism to generate E-Cad-85 from E-Cad-FL by treating lysates from SKOV3 cells and tumour tissue with this enzyme. Increased cytoplasmic localisation of $\beta$-Catenin in tumours with high E-Cad-85 expression corroborates that E-Cad-85 loses the binding site for $\beta$-Catenin after fragmentation, enabling tumour cluster formation and peritoneal dissemination.

Conclusions: Calpain-mediated E-Cad fragmentation appears to promote intraperitoneal EOC progression. Understanding these mechanisms might eventually lead to new tailored subtype-specific diagnostic and therapeutic interventions.

Epithelial ovarian cancer (EOC) represents the most lethal gynaecological malignancy with 239000 cases and 152000 deaths per year worldwide (Ferlay et al, 2014). About two-thirds of the patients are diagnosed in already advanced tumour stage (Nossov et al, 2008) with poor prognosis and a 5-year survival rate between $30 \%$ and $40 \%$ (Allemani et al, 2014). Major steps in ovarian cancer progression are peritoneal tumour dissemination and/or retroperitoneal lymph node involvement while distant metastases are rarely seen at first diagnosis (Prat and Oncology FCoG, 2014;
Gasimli et al, 2015). Over the past years, rising evidence has shown that distinct modes for progression may have prognostic implications for the patients. This has been taken into account in the most recent FIGO (International Federation of Gynecology and Obstetrics) staging classification for cancer of the ovary, fallopian tube and peritoneum published in 2014 (Prat and Oncology FCoG, 2014). In this revised classification, patients with retroperitoneal lymph node metastases and otherwise intrapelvic disease (FIGO stage IIIA1) are now distinguished from patients with macroscopic

\footnotetext{
*Correspondence: Professor S Mahner; E-mail: Sven.Mahner@med.uni-muenchen.de

${ }^{5}$ These authors contributed equally to this work.
}

Received 12 July 2015; revised 21 September 2015; accepted 19 November 2015; published online 12 January 2016

(c) 2016 Cancer Research UK. All rights reserved 0007-0920/16 
peritoneal extrapelvic disease (FIGO stage IIIB/C) that translated into significantly improved prognosis for FIGO stage IIIA1 in first explorative retrospective analyses (Pereira et al, 2015).

The molecular mechanisms underlying these different patterns of progression are poorly understood. E-Cadherin (E-Cad), a wellknown intercellular connecting protein maintaining the integrity of the conjunct cell-cell structure via homologue dimers, influences cellular adherence. Indispensable for E-Cad-mediated cellular function is $\beta$-Catenin enabling the connection to the cytoskeleton (Pokutta and Weis, 2007). For several adenocarcinomas, decreased E-Cad expression is correlated with an aggressive tumour type and poor survival (Faleiro-Rodrigues et al, 2004). In contrast to that, E-Cad expression was described to be overexpressed during transformation from normal ovarian surface epithelium to EOC (Dyck et al, 1996; Sundfeldt, 2003), although the role of E-Cad in metastases of EOC is inconclusive (Sundfeldt, 2003; Imai et al, 2004; Takai et al, 2014). Recent tumour biological investigations in breast cancer cell lines could demonstrate that E-Cad is processed by several proteases, including Calpain, resulting in fragments of different molecular size. These fragments exhibit divergent functions, including generation of tumour cell spheroids (Ye et al, 2013). As spheroid genesis and formation of lymphovascular emboli may promote tumour progression, this could represent a functional role for E-Cad in metastasis formation of ovarian cancer patients.

Therefore, it was hypothesised that E-Cad and its fragments may determine the route of tumour progression in ovarian cancer patients. Western blotting analyses, immunohistochemistry and calpain cleavage experiments were carried out to quantify the expression of E-Cad and its fragments in EOC with exclusive peritoneal tumour spread compared with tumours with retroperitoneal lymph node involvement. With the results, we aimed to better characterise their tumour growth pattern and identify possible new targets for therapeutic interventions.

\section{MATERIALS AND METHODS}

Patients. A total of 105 patients with available tumour tissue from primary advanced EOC treated at the University Medical Centre Hamburg-Eppendorf between 1995 and 2012 were included in the study. All patients underwent primary debulking surgery according to German guidelines (Wagner et al, 2013). Patient cohorts were classified with regard to their mode of tumour progression. A total of 41 patients had solely intraperitoneal tumour dissemination without lymph node involvement and a corresponding tumour stage pT3c, pN0 (intraperitoneal cohort). In contrast, 64 patients were diagnosed with lymph node metastases in tumour stage pT1a$3 \mathrm{c}, \mathrm{pN} 1$ (retroperitoneal cohort). Of these, 42 patients had positive lymph nodes with concurrent significant intraperitoneal tumour load (pT3c, pN1, two-sided group) and 22 patients with lymph node metastasis but only small peritoneal or pelvic tumour dissemination (pT1a-3b, pN1, predominant retroperitoneal group).

Detailed patient characteristics are presented in Table 1. All patients gave written informed consent to access their tissue and review their clinical records according to our investigational review board and ethics committee guidelines (no. 200814). Clinical parameters were retrieved from a detailed institutional database providing information on clinicopathological factors, histological classifications and therapeutic procedures. Clinical outcome of all patients was followed from date of first diagnosis until the end of 2014.

Tissue samples. Tissue samples were obtained intraoperatively and immediately stored in liquid nitrogen as fresh frozen samples. The histological characteristics of each sample were evaluated on cryo-cut and haematoxylin-eosin-stained sections. The tissue was tailored if necessary to obtain at least $70 \%$ invasive tumour cells in the sample used for protein extraction. Explorative western blotting analyses of different localisations from the same ovarian cancer were performed in parallel for a total of 10 tumour samples to test intratumour variability. In these investigations of the neighbouring tumour probes, no significant differences in terms of presence and intensity of the E-Cad-85 band was noted, confirming representativity of our results (Supplementary Figure S1).

Protein extractions. As described previously (Mahner et al, 2008), samples of approximately $100 \mathrm{mg}$ were cut from the tissue and pulverised using a micro-dismembrator (Braun-Melsungen, Melsungen, Germany) for $2 \mathrm{~min}$ and $45 \mathrm{~s}$ at 200 r.p.m. Proteins were lysed in ice-cold sample buffer ( $50 \mathrm{~mm}$ Tris $\mathrm{pH} 6.8,1 \%$ sodium dodecyl sulphate (SDS), $10 \%$ sucrose and $10 \mu \mathrm{lml}^{-1}$ protease inhibitor cocktail (Sigma, Taufkirchen, Germany)). The protein concentration was determined following standard protocols and using bovine serum albumin protein standards.

Western blotting analysis. For western blotting analysis, equal amounts of protein lysates $(20 \mu \mathrm{g})$ were analysed per well. The proteins were separated on a $10 \%$ SDS gel (11\% Glycerin, aqua dest., $10 \%$ SDS, Tris and $10 \%$ Acrylamid) and transferred to polyvinylidene difluoride membranes (Merck Millipore KGaA, Darmstadt, Germany). Later, the membranes were blocked with $5 \%$ nonfat dry milk (Bio-Rad Laboratories, Hercules, CA, USA) in TBST (TBS: $50 \mathrm{~mm}$ Tris, $150 \mathrm{~mm} \mathrm{NaCl}$, pH 7.4 with 10\% TWEEN 20 ), washed in TBST and incubated with the primary antibody. The monoclonal antibodies for E-Cad (24E10 no. 3195, 1:2000), $\beta$-Catenin (D10A8 no. 8480, 1:10000) and the polyclonal antiCalpain antibody (M-type no. 2539 1:1500; all purchased from Cell Signaling Technology, Leiden, The Netherlands) were diluted in $5 \% \mathrm{BSA}$ in TBST and incubated at $4{ }^{\circ} \mathrm{C}$ overnight. After incubation, membranes were washed in TBST and incubated with the corresponding secondary antibody (anti rabbit sc 2054, Santa Cruz Biotechnology, Heidelberg, Germany) in 1.5\% nonfat dry milk in TBST for $1 \mathrm{~h}$ at room temperature. Finally, after washing in TBST, the detection was carried out with the SuperSignal West Pico chemoluminescent kit (Thermo Scientific, Rockford, IL, USA) to visualise the protein expression on FUJI-super RX medical X-ray films (FUJI, Tokyo, Japan).

Band intensities were quantified by densitometry (GS-700 Imaging Densitometer, Bio-Rad, Munich, Germany). Protein lysates from the breast cancer cell line MCF7 were used as positive controls for E-Cad, from the ovarian cell line SKOV3 for $\beta$-Catenin and from the cervical cancer cell line HELA for Calpain. All detected bands of the investigated proteins except for the E-Cad fragment of $23 \mathrm{kDa}$ (E-Cad-23) were standardised with the positive controls, which were defined as $100 \%$. Protein expression levels were normalized to GAPDH (Santa Cruz FL 335; $1: 5000$ ). E-Cad23 was analysed semi-quantitatively and assigned to four categories (no expression $=0$, weak $=1$, moderate $=2$, strong $=3$, very strong $=4$ ) by two investigators.

Immunohistochemistry. Paraffin sections of tumour samples of 4-6 $\mu \mathrm{m}$ were deparaffinised in xylene and hydrated in a descending series of alcohol. After washing in aqua dest, the antigens were demasked over $20 \mathrm{~min}$ in $90^{\circ} \mathrm{C}$ heated citrate buffer $(10 \mathrm{~mm})$. Between two additional washing steps, the endogeneous peroxidase was blocked with $0.5 \% \mathrm{H}_{2} \mathrm{O}_{2}$. Later, sections were incubated at $4{ }^{\circ} \mathrm{C}$ overnight with the E-Cad $(1: 2000)$ or the $\beta$-Catenin $(1: 100)$ antibody, diluted 1:500 in antibody diluent (S0809, DAKO, Hamburg, Germany). Control sections were incubated simultaneously with an ISO-type control from DAKO (Rabbit IgG, No. X0903) at $4{ }^{\circ} \mathrm{C}$ overnight. After washing in TBS (5 mm Tris, $15 \mathrm{~mm} \mathrm{NaCl}, \mathrm{pH} 7.4$ ), the sections were incubated with the secondary antibody (DAKO ABC Elite Kit PK-6100) for $1 \mathrm{~h}$ at room temperature. After additional washing in TBS, E-Cad was visualised on tissue sections with the Peroxidase Substrate Kit 
Table 1. Patient characteristics according to the pattern of tumour dissemination

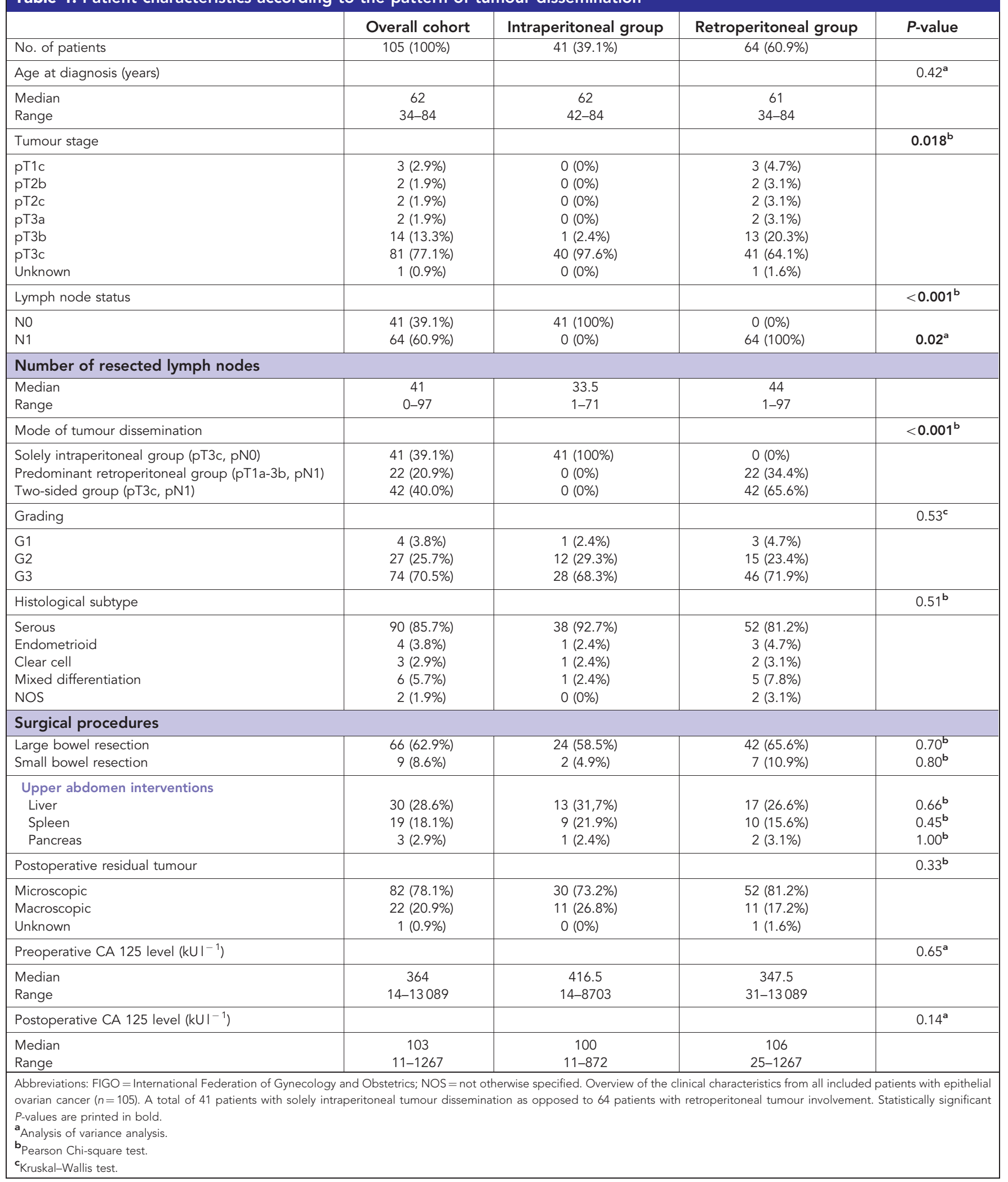

(DAB, SK-4100; DAKO), while cell nuclei were stained by haematoxylin (51275L, Sigma-Aldrich, St Louis, MO, USA). Finally, the sections were dehydrated in an ascending alcohol series and xylol.

E-Cad as well as $\beta$-Catenin expression and their localisation was analysed by two independent investigators evaluating scores for staining intensity (SI, $0=$ negative, $1=$ weak, $2=$ slightly positive, $3=$ strong expression) and percentage of positive cells (PP, $1=<50 \%, 2=\geqslant 50 \%$ ). To objectify cytoplasmic expression, SI was applied while membranous expression was the product of SI multiplied by PP.

Calpain assay. The ovarian cancer cell line SKOV3 was cultivated in McCoy's 5A Medium (GE Healthcare, PAA Laboratories, 
Pasching, Austria) with 10\% foetal bovine serum (PAA Laboratories) till $90 \%$ confluency. After washing in PBS, the cells were scratched with a cell scraper and homogenised in extraction buffer (50 mм HEPES (ph 7.5), 1 mм EGTA, $50 \mathrm{~mm} \mathrm{KCl,} 2 \mathrm{~mm} \mathrm{MgCl}$, $5 \mathrm{~mm}$ Mercaptoethanol and $1 \mu \mathrm{l}$ protease inhibitor cocktail without Leupeptin (P8849, Sigma-Aldrich) per $100 \mu$ l extraction-buffer). The solution was stored $30 \mathrm{~min}$ on ice before centrifugation at $1000 \mathrm{~g}$ for $10 \mathrm{~min}$ at $4{ }^{\circ} \mathrm{C}$. Equal amounts of protein lysates were incubated with different treatments for $60 \mathrm{~min}$ at $30^{\circ} \mathrm{C}$ : without adjunct, with $1 \mathrm{~mm} \mathrm{CaCl}$, with $1 \mathrm{mM} \mathrm{CaCl}_{2}$ and $0.3 \mathrm{U}$ (Units) $\mu$-Calpain (C6108, Sigma-Aldrich), and with $1 \mathrm{~mm} \mathrm{CaCl}_{2}, 0.3 \mathrm{U}$ $\mu$-Calpain and $10 \mathrm{~mm}$ Calpeptin (CAS 117591-20-5, Calbiochem, Darmstadt, Germany). Treatment was stopped by addition of $2 \times$ sample buffer (62.5 mm Tris-HCL (pH 6.8), 4\% SDS, 10\% glycerol) and 10 -min incubation at $100{ }^{\circ} \mathrm{C}$. In addition to SKOV3, three exemplary lysates of tumour tissue from the intraperitoneal group (T5213, T6362, T6570) were treated in the same way. After treatment, the protein lysates were investigated in western blotting analysis with the same monoclonal E-Cad antibody (24E10).

Statistical analysis. The expression levels of E-Cad and $\beta$-Catenin bands were compared between the different cohorts by ANOVA analysis, as well as post hoc with the Least Significant Difference Test. Fragments of E-Cad were correlated by Spearman Rho among themselves and with the protease Calpain, as well as with $\beta$ Catenin. Correlation coefficients were assessed as significantly strong according to the notes on medical statistics (Bland and Altman, 1994). Expression scores from immunohistochemical analyses were compared by Student's $t$-test. Kaplan-Meier estimates and log-rank test were carried out to illustrate and compare progression-free survival (PFS) and overall survival (OS). In this context, patients were categorised in tertiles according to their levels of E-Cad-85 expression. The associated hazard ratios were determined by Cox regression. $P$-values $\leqslant 0.05$ were considered to be statistically significant. All statistical analyses were carried out with SPSS Statistics version 22 for Windows (IBM, Armonk, NY, USA). Figures were edited with Stata 13.1 (StataCorp, College Station, TX, USA, 2014).

\section{RESULTS}

A total of 105 patients were included in this study, 41 (39.1\%) with only intraperitoneal and $64(61.0 \%)$ with retroperitoneal tumour growth. Patient characteristics were well balanced between both groups except for the obvious difference in tumour stage as well as significantly more resected lymph nodes in patients with retroperitoneal tumour dissemination (median 44 vs 34 lymph nodes, $P=0.02$; Table 1$)$.

Ovarian cancer with solely peritoneal metastases exhibits high levels of E-Cad fragments. In western blotting analysis, fulllength E-Cad (E-Cad FL) at $120 \mathrm{kDa}$ was detected in almost all investigated tumour samples. No significant differences between the solely intraperitoneal group compared with tumours with retroperitoneal involvement were noted (median 1.57 vs 1.20, $P=0.21$; Figures 1 and $2 \mathrm{~A}$ ).

In addition to the expected band at $120 \mathrm{kDa}$, further signals for fragments between 70 and $100 \mathrm{kDa}$ were detected by the anti-E-Cad monoclonal antibody. Of these, the $85-\mathrm{kDa}$ fragment (E-Cad-85) exhibited the strongest intensity, while fragments at $100 \mathrm{kDa}$ were only very weak and not reliably detectable (Figure 1). E-Cad-85 showed a significantly higher expression in the group of exclusive peritoneal metastases compared with the group with retroperitoneal metastasis (median 0.48 vs $0.31, \quad P=0.01$; Figure $2 \mathrm{~B})$. An additional signal at $23 \mathrm{kDa}$ (E-Cad-23) could be detected representing a further, seemingly cytoplasmic fragment of E-Cad according to the cytoplasmic binding site of the E-Cad antibody 24E10 (Figure 1). Expression differences between the intraperitoneal and the retroperitoneal group of E-Cad-23 were non-significant (median 2.00 vs 1.00, $P=0.07$; Figure 2C).

Within the overall cohort, E-Cad-FL expression correlated significantly with E-Cad-85 $(r=0.80 ; P<0.0001)$ and E-Cad-23 $(r=0.44 ; \quad P<0.0001$, Table $2 \mathrm{~A}$, Figure 1) with comparable interactions in tumours with solely peritoneal metastases (E-CadFL and E-Cad-85 $r=0.79 ; P<0.0001$, E-Cad-FL and E-Cad-23 $r=0.49 ; P=0.001$, Table $2 \mathrm{~B})$ as well as in tumours with

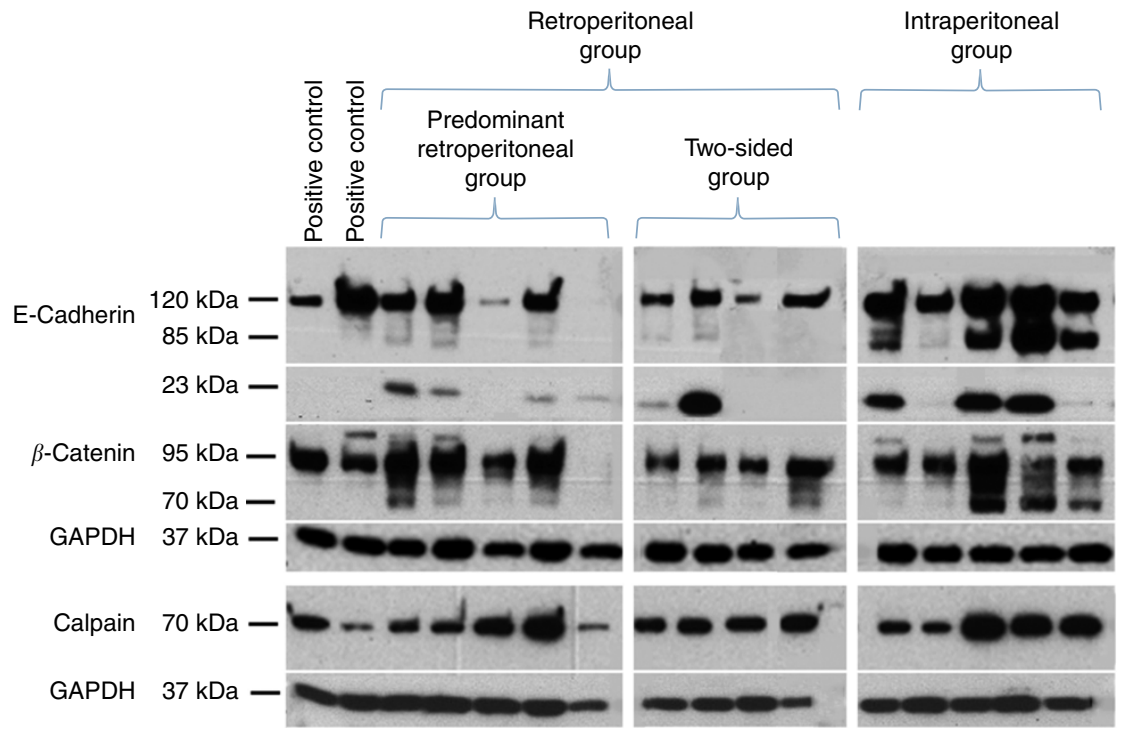

Figure 1. Expression of E-Cadherin, $\beta$-Catenin and Calpain according to the mode of tumour progression. Western blotting analysis showing representative expression of E-Cadherin (E-Cad-85 and E-Cad-23), $\beta$-Catenin and Calpain in the different types of tumour dissemination. Protein lysates from the breast cancer cell line MCF7 were used as positive controls for E-Cadherin, from the ovarian cell line SKOV3 for $\beta$-Catenin and from the cervical cancer cell line HELA for Calpain. Equal amounts of protein lysate $(20 \mu \mathrm{g})$ were loaded per well. As a loading control, GAPDH protein expression was detected. 

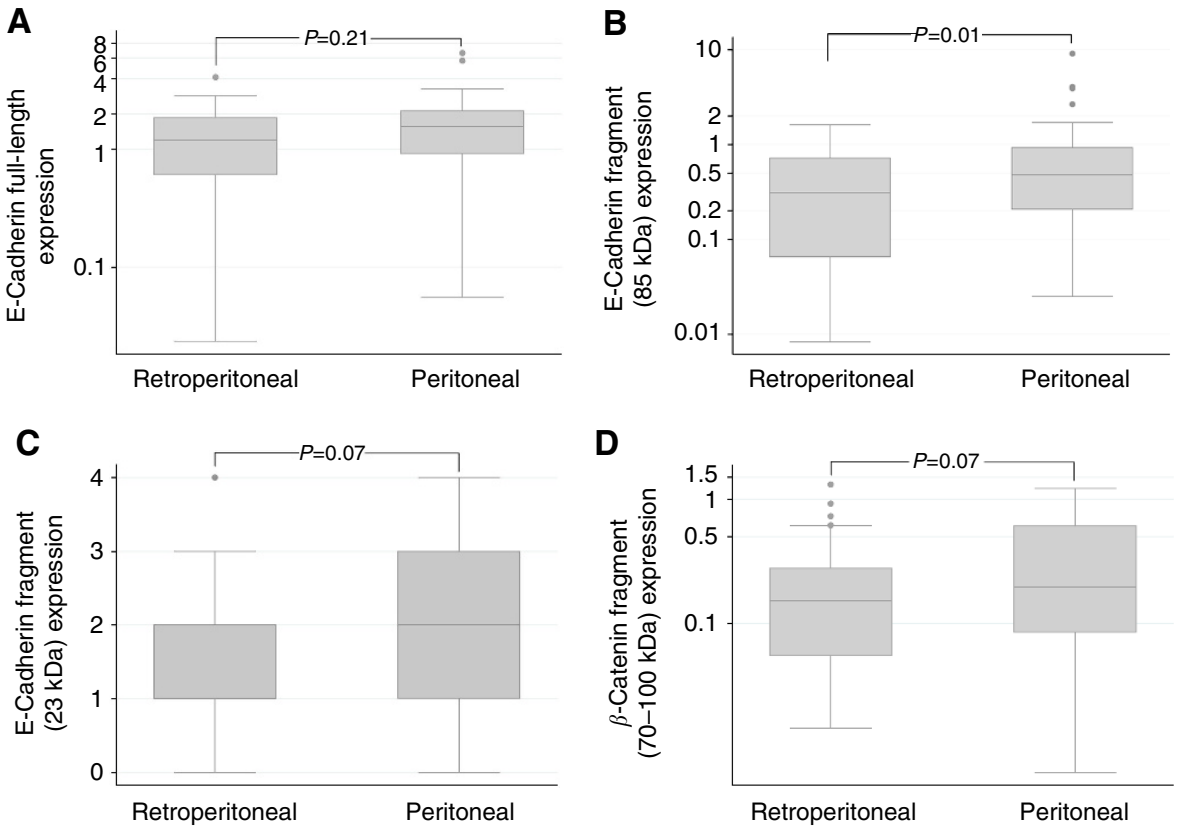

Figure 2. Quantitative expression of E-Cad-FL, E-Cad-85, E-Cad-23 and $\beta$-Catenin fragments according to the mode of tumour progression. (A) E-Cad-FL was expressed in all investigated tumour samples but without significant differences between the two groups ( $P=0.21)$. (B) E-Cad-85 with significantly higher expression in the group of solely intraperitoneal metastases compared with retroperitoneal metastases $(P=0.01)$. (C, D) E-Cad-23 and $\beta$-Catenin (70-100 kDa) did not exhibit significant differences in the expression values between both groups (each with $P=0.07)$.

Table 2A. Correlation of the protein expression among each other in the overall cohort $(n=105)$

\begin{tabular}{|c|c|c|c|c|}
\hline & E-Cad-85 & E-Cad-23 & Calpain & $\beta$-Catenin \\
\hline E-Cad-85 & - & $\begin{array}{c}r=0.47 \\
P<0.0001\end{array}$ & $\begin{aligned} r & =0.30 \\
P & =0.002\end{aligned}$ & $\begin{array}{l}r=0.17 \\
P=0.09\end{array}$ \\
\hline E-Cad-23 & $\begin{array}{c}r=0.47 \\
P<0.0001\end{array}$ & - & $\begin{array}{l}r=0.20 \\
P=0.04\end{array}$ & $\begin{array}{l}r=0.24 \\
P=0.01\end{array}$ \\
\hline Calpain & $\begin{aligned} r & =0.30 \\
P & =0.002\end{aligned}$ & $\begin{array}{l}r=0.20 \\
P=0.04\end{array}$ & - & $\begin{array}{c}r=0.35 \\
P=0.0003\end{array}$ \\
\hline E-Cad-FL & $\begin{array}{c}r=0.80 \\
P<0.0001\end{array}$ & $\begin{array}{c}r=0.44 \\
P<0.0001\end{array}$ & $\begin{aligned} r & =0.24 \\
P & =0.016\end{aligned}$ & $\begin{aligned} r & =0.32 \\
P & =0.001\end{aligned}$ \\
\hline
\end{tabular}

retroperitoneal involvement (E-Cad-FL and E-Cad-85 $r=0.80$ $P<0.001$, E-Cad-FL and E-Cad-23 $r=0.37 ; P=0.003$, Table 2C).

A significant correlation between E-Cad-23 and E-Cad-85 could be demonstrated in the overall cohort $(r=0.47 ; P<0.0001$, Table 2A, Figure 1) being even stronger in the cohort of solely peritoneal metastases $(r=0.56 ; P<0.0001$, Table $2 \mathrm{~B}$, Figure 1$)$ and weaker in tumours with retroperitoneal metastases $(r=0.40$; $P=0.001$, Table 2C).

For $\beta$-Catenin, fragments between 70 and $90 \mathrm{kDa}$ in addition to full-length $\beta$-Catenin were detected. Yet, the expression values were not statistically different between the two groups (median 0.20 vs $0.15, P=0.07$; Figure 2D). Accordingly, $\beta$-Catenin did not correlate with E-Cad-85 ( $\beta$-Catenin and E-Cad-85 $\quad r=0.17$ $P=0.09)$ and only weakly with E-Cad-23 in the overall cohort ( $\beta$-Catenin and E-Cad-23 $r=0.24 ; P=0.01$; Table 2A).

Expression of Calpain correlates with E-Cad-85 and E-Cad-23. As E-Cad-85 expression values differed significantly among tumours with different metastatic patterns, we were interested in the formation and functional significance of this fragment. Calpain is an intracellular protease, which cleaves E-Cad in the area of the
Table 2B. Correlation of the protein expression among each other in the group of solely intraperitoneal metastases $(n=41)$

\begin{tabular}{|c|c|c|c|c|}
\hline & E-Cad-85 & E-Cad-23 & Calpain & $\beta$-Catenin \\
\hline E-Cad-85 & - & $\begin{array}{c}r=0.56 \\
P=0.0001\end{array}$ & $\begin{aligned} r & =0.48 \\
P & =0.002\end{aligned}$ & $\begin{array}{l}r=0.21 \\
P=0.19\end{array}$ \\
\hline E-Cad-23 & $\begin{array}{c}r=0.56 \\
P=0.0001\end{array}$ & - & $\begin{aligned} r & =0.31 \\
P & =0.048\end{aligned}$ & $\begin{array}{l}r=0.28 \\
P=0.08\end{array}$ \\
\hline Calpain & $\begin{aligned} r & =0.48 \\
P & =0.002\end{aligned}$ & $\begin{aligned} r & =0.31 \\
P & =0.048\end{aligned}$ & - & $\begin{array}{l}r=0.19 \\
P=0.24\end{array}$ \\
\hline E-Cad-FL & $\begin{array}{c}r=0.79 \\
P<0.0001\end{array}$ & $\begin{aligned} r & =0.49 \\
P & =0.001\end{aligned}$ & $\begin{aligned} r & =0.51 \\
P & =0.001\end{aligned}$ & $\begin{array}{l}r=0.21 \\
P=0.20\end{array}$ \\
\hline
\end{tabular}

Table 2C. Correlation of the protein expression among each other in the group of retroperitoneal involvement $(n=64)$

\begin{tabular}{|c|c|c|c|c|}
\hline & E-Cad-85 & E-Cad-23 & Calpain & $\beta$-Catenin \\
\hline E-Cad-85 & - & $\begin{aligned} r & =0.40 \\
P & =0.001\end{aligned}$ & $\begin{array}{l}r=0.12 \\
P=0.37\end{array}$ & $\begin{array}{l}r=0.07 \\
P=0.60\end{array}$ \\
\hline E-Cad-23 & $\begin{aligned} r & =0.40 \\
P & =0.001\end{aligned}$ & - & $\begin{array}{l}r=0.08 \\
P=0.55\end{array}$ & $\begin{array}{l}r=0.19 \\
P=0.14\end{array}$ \\
\hline Calpain & $\begin{array}{l}r=0.12 \\
P=0.37\end{array}$ & $\begin{array}{l}r=0.08 \\
P=0.55\end{array}$ & - & $\begin{aligned} r & =0.40 \\
P & =0.002\end{aligned}$ \\
\hline E-Cad-FL & $\begin{array}{l}r=0.80 \\
P<0.001\end{array}$ & $\begin{aligned} r & =0.37 \\
P & =0.003\end{aligned}$ & $\begin{array}{c}r=-0.01 \\
P=0.99\end{array}$ & $\begin{array}{c}r=0.33 \\
P=0.008\end{array}$ \\
\hline
\end{tabular}

$P=P$-value; correlation coefficient: $r=$ Spearman Rho. Bold values indicate statistically significant $P$-values.

amino-acid Pro780 at the cytoplasmic domain, leading to two fragments of 85 and $20-25 \mathrm{kDa}$ (Supplementary Figure S2). Based on the hypothesis that the $85-\mathrm{kDa}$ fragment found in our tumours is generated by calpain activity, we performed western blotting 
analysis of our total cohort using an anti-calpain antibody. Within the overall cohort, Calpain expression correlated significantly with E-Cad-85 fragments $(r=0.30 ; P=0.002$, Table 2A, Figure 1$)$, which was more pronounced in the cohort of solely peritoneal metastases $(r=0.48 ; P=0.002$, Table $2 \mathrm{~B}$, Figure 1$)$. In addition, Calpain expression correlated with E-Cad-23 levels in the total cohort $(r=0.20 ; P=0.04)$ and tumours showing exclusively intraperitoneal spread $(r=0.31 ; P=0.048$, Tables $2 \mathrm{~A}$ and $2 \mathrm{~B})$, which might link cleavage by Calpain with these E-Cad fragments. Contrarily, this correlation was not present in tumours with retroperitoneal involvement (E-Cad-85 and Calpain $r=0.12$, $P=0.37$; E-Cad-23 and Calpain $r=0.08, P=0.55$, Table 2C).

Evaluation of possible functional implications of the E-Cad-85 fragment. Human E-Cad consists of an extracellular part, which exhibits five domains, a trans-membranous and a highly conserved cytoplasmic domain. Via the cytoplasmic domain, E-Cad is linked to the two connection proteins p120 catenin (p120ctn) and $\beta$ catenin establishing the intracellular anchoring. These protein complexes coordinate the organisation of the cell in basal and apical orientation guaranteeing a stable configuration of the cellcell contact (Supplementary Figure S2; Pokutta and Weis, 2007).

The applied E-Cad antibody (24E10) detects E-Cad intracellularly in the area of the amino-acid sequence Pro780 of E-Cad (Supplementary Figure S2, labelled as 24E10-binding site), which is concomitantly the cleavage domain of Calpain. Supplementary Figure S2 illustrates our hypothesised position of the E-Cad-85 fragment in relation to the cell membrane leading to a large extracellular region of the protein with the 24E10-binding site intracellularly at the proximal end of the fragment. In this distribution, E-Cad-85 is characterised by the loss of the cytoplasmic-binding domain to $\beta$-Catenin, so that E-Cad- 85 is not able to bind to the cytoskeleton. Consequently, a stable configuration of the conjunct cell-cell structure cannot be guaranteed.

To test this biological hypothesis, paraffin-embedded sections of tumour samples were evaluated by immunohistochemistry for E-Cad and $\beta$-Catenin ( $n=40$; solely intraperitonal $n=15$, retroperitoneal $n=25$ ). A strong membraneous E-Cad signal and a positive but weaker cytosolic expression was detected in all the investigated tumour sections. Membranous expression was significantly higher in patients with retroperitoneal involvement compared with patients with solely intraperitoneal tumour dissemination (mean membranous expression score 2.31 vs. 3.10, $P=0.02$, Figure $3 \mathrm{~A}$ ). $\beta$-Catenin was evaluated with regard to localisation and staining intensity in two groups comparing patients with high E-Cad-85 expression (above median) in western blotting to patients with low E-Cad-85 (below median) expression values. In patients with high E-Cad-85 following suspected E-Cad fragmentation, cytoplasmic $\beta$-Catenin expression was significantly higher compared with patients with low E-Cad-85 levels (mean cytoplasmic expression score 1.80 vs. $0.80, P<0.001$, Figure $3 \mathrm{~B}$ ) while membranous staining was not statistically different between both groups (mean membranous expression score 2.85 vs. 3.31, $P=0.18$, Figure 3B).

To evaluate a possible prognostic significance of E-Cad-85, we stratified the overall cohort expression in tertiles according to their E-Cad-85 expression levels resulting in three groups with low $(n=72)$, moderate $(n=27)$ and high $(n=4)$ E-Cad-85 expression. Patients with both low and moderate E-Cad-85 expression had a median PFS of 23 months, respectively, which was significantly longer compared with patients with high E-Cad-85 expression and a PFS of 8 months (Supplementary Figure S3A; moderate $v s$ high, $P=0.003$; low $v s$ high, $P=0.007$ ). In accordance, median $\mathrm{OS}$ of patients with low and moderate E-Cad-85 expression was significantly better with 50 and 53 months, respectively, compared with 17 months in that of patients with high E-Cad-85 expression (Supplementary Figure S3B; moderate vs high, $P=0.04$; low vs high, $P=0.001)$.

Cleavage of E-Cad-FL to E-Cad-85 fragments by Calpain in lysates of SKOV3 cells and ovarian tumour tissue. In western blotting analysis of SKOV3 ovarian tumour cells, E-Cad FL was almost exclusively detected. Following Calpain treatment of the SKOV3 proteins, a strong E-Cad-85 fragment appeared in addition to E-Cad FL. This Calpain cleavage could be inhibited by addition of the Calpain inhibitor Calpeptin (Figure 4A). In three exemplary lysates of tumour tissue from the intraperitoneal group (Figure 4B: T5213, Figure 4C: T6362, Figure 4D: T6570), the same trend could be confirmed, showing increased E-Cad-85 and decreased E-Cad FL levels after calpain cleavage, which was partly inhibited by calpeptin. These results correspond to the observed correlation of the Calpain expression with E-Cad fragments in EOC as shown in western blotting analysis.
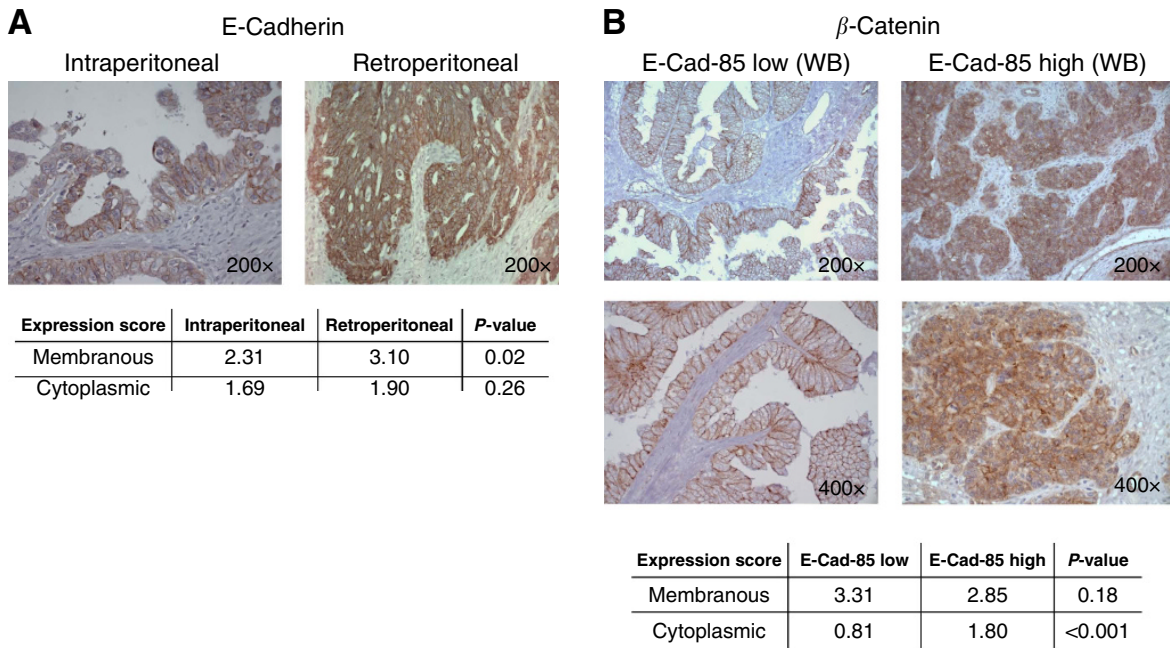

Figure 3. Immunohistochemistry for E-Cad and $\beta$-Catenin expression pattern in ovarian cancer tissue samples. (A) Membranous E-Cad expression was significantly higher in tumour samples from patients of the retroperitoneal group compared with patients of the intraperitoneal group, while cytoplasmic E-Cad expression did not differ. (B) Cytoplasmic expression of $\beta$-Catenin was significantly higher in tumour tissue from patients with high E-Cad-85 compared with patients with low E-Cad-85 expression levels that were previously estimated by western blotting. No significant difference in membranous $\beta$-Catenin expression was noted. 
A
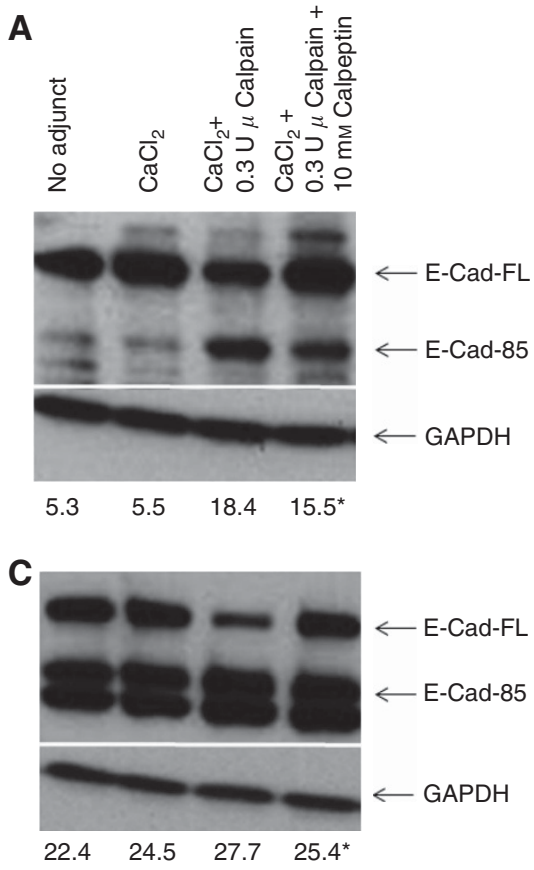

B

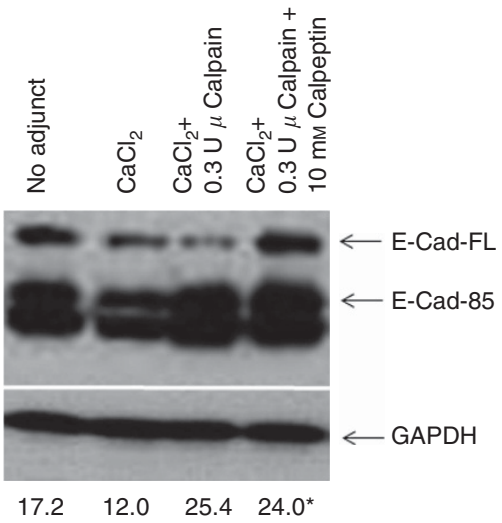

D

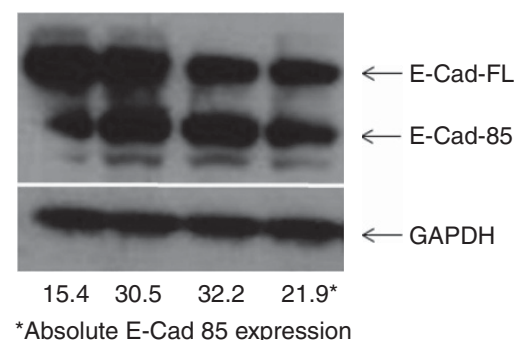

Figure 4. Expression of E-Cad-FL and E-Cad-85 following treatment with Calpain and Calpeptin in SKOV3-WT cells and tumour tissue lysates. (A) SKOV3-WT ovarian tumour cells exhibited E-Cad-85 in addition to E-Cad-FL after Calpain treatment, while E-Cad-85 expression was decreased following addition of Calpeptin. (B-D) Treatment of three tumour tissue lysates ((B) T5213, (C) T6362 and (D) T6570) revealed the same trend with Calpain-promoted increased expression of E-Cad-85 in contrast to reduced E-Cad fragmentation following Calpeptin treatment.

\section{DISCUSSION}

In this study of 105 patients with advanced ovarian cancer, we could observe a potential role for Calpain-mediated E-Cad fragments in promotion of intraperitoneal tumour progression.

For most tumour entities, decreased expression of adhesion proteins is associated with a more invasive growth pattern, lower histological differentiation and metastatic disease (FaleiroRodrigues et al, 2004). In terms of ovarian cancer, conflicting results regarding E-Cad have been published. E-Cad is initially upregulated when normal ovarian surface cells are transformed to ovarian cancer cells. During this process, mesothelial-like, $\mathrm{N}$-Cadherin-positive cells transform to epithelial, E-Cad-positive tumour cells (Sundfeldt, 2003). Over the following steps towards metastatic EOC, a transient impact of E-Cad has been proposed, mainly based on immunohistochemical investigations (Sundfeldt, 2003; Imai et al, 2004; Takai et al, 2014). In the present study, E-Cad expression was detected in EOC with solely intraperitoneal tumour dissemination as well as in EOC with retroperitoneal involvement. At first glance, this was not anticipated as these tumours are characterised by agile, independent tumour cells and cell clusters and not by strong cell-cell interactions within the tumour. However, based on our western blotting results for different E-Cad fragments we hypothesised an important role of E-Cad-85 in defining the mode of tumour progression. Accounting for the cytoplasmic-binding site of the E-Cad antibody, the calpain cleavage site and the size of the fragment, E-Cad-85 might miss most of the intracellular domain, including the $\beta$-catenin-binding site (Supplementary Figure S2).

In 2013, Ye et al (2013) described a comparable distribution of E-Cad fragments in the breast cancer cell line MARY-X. Their experiments revealed that Calpain-generated E-Cad fragments at $100 \mathrm{kDa}$ are spread all over the tumour cells instead of being localised in adherent junctions of cell-cell interactions. This non-directional distribution enables the formation of tumour-cell clusters and E-Cad-positive spheroids potentially favouring metastases (Ye et al, 2013). E-Cad-85 seems to be further processed losing the $\mathrm{N}$-terminus but retaining the ability to peritoneal progression in tumour environment, possibly by spheroid genesis as previously described for the E-Cad fragment at $100 \mathrm{kDa}$.

Calpain was identified as an intracellular protease that accurately cleaves E-Cadherin in the area of the amino-acid sequence Pro780 (Rios-Doria et al, 2003) and its expression correlated significantly with the E-Cad-85 and E-Cad-23 fragments. Following Calpain treatment in vitro, stronger E-Cad-85 fragments appeared in SKOV3 lysates. The same trend in lysates of exemplary tumour tissue from the intraperitoneal group corroborates the assumption that Calpain cleaves E-Cad FL to its fragments in EOC. E-Cad-85 therefore loses the binding site to $\beta$-Catenin enabling tumour cluster formation. This possible mechanism could be supported by immunohistochemical analyses for $\beta$-Catenin. In these experiments, cytoplasmic expression of $\beta$-Catenin was more pronounced in tumours with high E-Cad-85 expression seemingly attributed to the released connection between membranous E-Cad-85 and $\beta$-Catenin following E-Cad cleavage. Accordingly, Salehin et al (2011) observed decreased Calpain expression levels with increasing lymph node status in immunohistochemical investigations.

From our present results, it can be proposed that intraperitoneal metastases are promoted by Calpain-generated E-Cad-85. A different example of posttranslational E-Cad modification influencing tumour growth in ovarian cancer has been previously shown (Symowicz et al, 2007). In these experiments, exogenous MMP-9 catalysed E-Cad ectodomain shedding, which enhanced survival and migration of tumour cells by enabling multicellular aggregates in ascites (Symowicz et al, 2007). Based on these results and our present data, posttranslational changes might explain the divergent roles of E-Cad in benign conditions such as acute pancreatitis (Mayerle et al, 2005) as well as in inflammatory breast cancer (Ye et al, 2013), prostate cancer (Rios-Doria et al, 2003) and in EOC (Symowicz et al, 2007). 
To date, E-Cad function has been investigated in early and advanced EOC but without accounting for different modes of metastases. Progression of advanced EOC has been explained by epithelial-mesenchymal transition (Strauss et al, 2011), which, however, conflicts with the described increased E-Cad expression in metastases (Imai et al, 2004). For peritoneal tumour spread, the formation of spheroids seems to be beneficial as these cell clusters can passively be carried by the peritoneal fluid and may overcome apoptosis and anoikis (Lengyel, 2010; Carduner et al, 2014). Our hypothesis attributes an important role to E-Cad-85 fragments for EOC progression and enables further understanding of the molecular process of peritoneal metastasis. In addition to quantitative analysis, we were able to draw functional conclusions from the expression of E-Cad, its fragments and their relation to $\beta$-Catenin as well as Calpain regarding the mode of tumour progression. These promising results are, however, based on western blotting and immunohistochemical investigations of retrospectively analysed fresh frozen tumour samples. Although the number of analysed samples from 105 patients is considerably large, it is important to highlight the explorative analytical approach. Although the elucidated negative prognostic impact of E-Cad-85 might represent a link to the observed worse prognosis of EOC with peritoneal tumour spread compared with tumours solely lymph node metastases, future studies with larger, ideally prospectively collected cohorts will be necessary to substantiate our results. Investigating the protease Calpain and E-Cad fragments as potential therapeutic targets might also be of high clinical interest as understanding the mechanisms for EOC tumour progression will open diagnostic and therapeutic interventions tailored for specific tumour types.

\section{CONFLICT OF INTEREST}

The authors declare no conflict of interest.

\section{REFERENCES}

Allemani C, Weir HK, Carreira H, Harewood R, Spika D, Wang XS, Bannon F, Ahn JV, Johnson CJ, Bonaventure A, Marcos-Gragera R, Stiller C, Azevedo ESG, Chen WQ, Ogunbiyi OJ, Rachet B, Soeberg MJ, You H, Matsuda T, Bielska-Lasota M, Storm H, Tucker TC, Coleman MP. the CWG (2014) Global surveillance of cancer survival 1995-2009: analysis of individual data for 25676887 patients from 279 population-based registries in 67 countries (CONCORD-2). Lancet 385(9972): 977-1010.

Bland JM, Altman DG (1994) Correlation, regression, and repeated data BMJ 308(6933): 896.

Carduner L, Picot CR, Leroy-Dudal J, Blay L, Kellouche S, Carreiras F (2014) Cell cycle arrest or survival signaling through alphav integrins, activation of PKC and ERK1/2 lead to anoikis resistance of ovarian cancer spheroids. Exp Cell Res 320(2): 329-342.

Dyck HG, Hamilton TC, Godwin AK, Lynch HT, Maines-Bandiera S, Auersperg N (1996) Autonomy of the epithelial phenotype in human ovarian surface epithelium: changes with neoplastic progression and with a family history of ovarian cancer. Int J Cancer 69(6): 429-436.

Faleiro-Rodrigues C, Macedo-Pinto I, Pereira D, Ferreira VM, Lopes CS (2004) Association of E-cadherin and beta-catenin immunoexpression with clinicopathologic features in primary ovarian carcinomas. Hum Pathol 35(6): 663-669.

Ferlay J, Soerjomataram I, Dikshit R, Eser S, Mathers C, Rebelo M, Parkin DM, Forman D, Bray F (2014) Cancer incidence and mortality worldwide: sources, methods and major patterns in GLOBOCAN 2012. Int J Cancer 136(5): E359-E386.

Gasimli K, Braicu EI, Richter R, Chekerov R, Sehouli J (2015) Prognostic and predictive value of the peritoneal cancer index in primary advanced epithelial ovarian cancer patients after complete cytoreductive surgery: study of tumor bank ovarian cancer. Ann Surg Oncol 22(8): 2729-2737.
Imai T, Horiuchi A, Shiozawa T, Osada R, Kikuchi N, Ohira S, Oka K, Konishi I (2004) Elevated expression of E-cadherin and alpha-, beta-, and gamma-catenins in metastatic lesions compared with primary epithelial ovarian carcinomas. Hum Pathol 35(12): 1469-1476.

Lengyel E (2010) Ovarian cancer development and metastasis. Am J Pathol 177(3): 1053-1064.

Mahner S, Baasch C, Schwarz J, Hein S, Wolber L, Janicke F, Milde-Langosch $\mathrm{K}$ (2008) C-Fos expression is a molecular predictor of progression and survival in epithelial ovarian carcinoma. Br J Cancer 99(8): 1269-1275.

Mayerle J, Schnekenburger J, Kruger B, Kellermann J, Ruthenburger M, Weiss FU, Nalli A, Domschke W, Lerch MM (2005) Extracellular cleavage of E-cadherin by leukocyte elastase during acute experimental pancreatitis in rats. Gastroenterology 129(4): 1251-1267.

Nossov V, Amneus M, Su F, Lang J, Janco JM, Reddy ST, Farias-Eisner R (2008) The early detection of ovarian cancer: from traditional methods to proteomics. Can we really do better than serum CA-125? Am J Obstet Gynecol 199(3): 215-223.

Pereira A, Perez-Medina T, Magrina JF, Magtibay PM, Rodriguez-Tapia A, Peregrin I, Mendizabal E, Ortiz-Quintana L (2015) International Federation of gynecology and obstetrics staging classification for cancer of the ovary, fallopian tube, and peritoneum: estimation of survival in patients with node-positive epithelial ovarian cancer. Int J Gynecol Cancer 25(1): 49-54.

Pokutta S, Weis WI (2007) Structure and mechanism of cadherins and catenins in cell-cell contacts. Annu Rev Cell Dev Biol 23: 237-261.

Prat J. Oncology FCoG (2014) Staging classification for cancer of the ovary, fallopian tube, and peritoneum. Int J Gynaecol Obstet 124(1): 1-5.

Rios-Doria J, Day KC, Kuefer R, Rashid MG, Chinnaiyan AM, Rubin MA, Day ML (2003) The role of calpain in the proteolytic cleavage of E-cadherin in prostate and mammary epithelial cells. J Biol Chem 278(2): 1372-1379.

Salehin D, Fromberg I, Haugk C, Dohmen B, Georg T, Bohle RM, Bauerschlag D, Thill M, Friedrich M (2011) Immunhistochemical analysis for expression of calpain 1, calpain 2 and calpastatin in ovarian cancer. Eur J Gynaecol Oncol 32(6): 628-635.

Strauss R, Li ZY, Liu Y, Beyer I, Persson J, Sova P, Moller T, Pesonen S, Hemminki A, Hamerlik P, Drescher C, Urban N, Bartek J, Lieber A (2011) Analysis of epithelial and mesenchymal markers in ovarian cancer reveals phenotypic heterogeneity and plasticity. PLoS One 6(1): e16186.

Sundfeldt K (2003) Cell-cell adhesion in the normal ovary and ovarian tumors of epithelial origin; an exception to the rule. Mol Cell Endocrinol 202(1-2): 89-96.

Symowicz J, Adley BP, Gleason KJ, Johnson JJ, Ghosh S, Fishman DA, Hudson LG, Stack MS (2007) Engagement of collagen-binding integrins promotes matrix metalloproteinase-9-dependent E-cadherin ectodomain shedding in ovarian carcinoma cells. Cancer Res 67(5): 2030-2039.

Takai M, Terai Y, Kawaguchi H, Ashihara K, Fujiwara S, Tanaka T, Tsunetoh S, Tanaka Y, Sasaki H, Kanemura M, Tanabe A, Ohmichi M (2014) The EMT (epithelial-mesenchymal-transition)-related protein expression indicates the metastatic status and prognosis in patients with ovarian cancer. J Ovarian Res 7: 76.

Wagner U, Harter P, Hilpert F, Mahner S, Reuss A, du Bois A, Petru E, Meier W, Ortner P, Konig K, Lindel K, Grab D, Piso P, Ortmann O, Runnebaum I, Pfisterer J, Luftner D, Frickhofen N, Grunwald F, Maier BO, Diebold J, Hauptmann S, Kommoss F, Emons G, Radeleff B, Gebhardt M, Arnold N, Calaminus G, Weisse I, Weis J, Sehouli J, Fink D, Burges A, Hasenburg A, Eggert C (2013) S3-guideline on diagnostics, therapy and follow-up of malignant ovarian tumours: short version 1.0 - AWMF registration number: 032/035OL, June 2013. Geburtshilfe Frauenheilkund 73(9): 874-889.

Ye Y, Tian H, Lange AR, Yearsley K, Robertson FM, Barsky SH (2013) The genesis and unique properties of the lymphovascular tumor embolus are because of calpain-regulated proteolysis of E-cadherin. Oncogene 32(13): $1702-1713$.

This work is published under the standard license to publish agreement. After 12 months the work will become freely available and the license terms will switch to a Creative Commons AttributionNonCommercial-Share Alike 4.0 Unported License.

Supplementary Information accompanies this paper on British Journal of Cancer website (http://www.nature.com/bjc) 